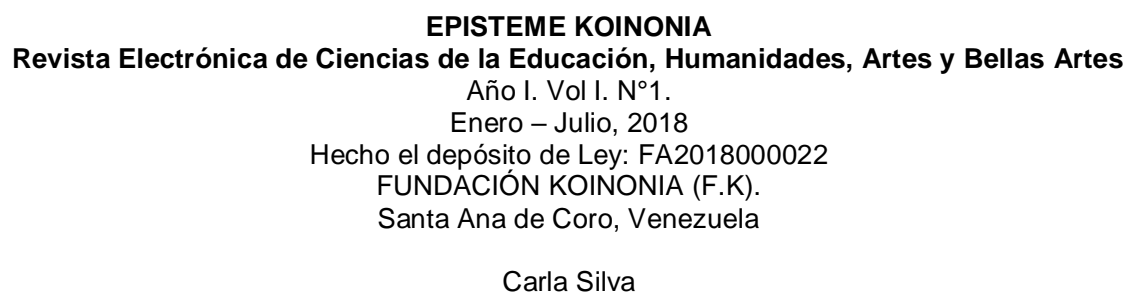

\title{
Formación integral para facilitadores
}

\section{Integral formation for facilitators}

\author{
Carla Silva \\ Carla_186_9@hotmail.com \\ Universidad Politécnica Territorial del Estado Barinas José Félix Rivas, Barinas \\ Venezuela
}

Recibido: 25 de octubre de 2017

Aprobado: 30 de noviembre de 2017

\begin{abstract}
RESUMEN
El principal objetivo de este trabajo ha sido la elaboración de una estrategia metodológica para el desarrollo del proceso de formación pedagógico-didáctico para facilitadores, partiendo de que las Universidades pretenden vincular a su plantilla de catedráticos a personas que se destacan en su ejercicio profesional y que, por ende, considera que tienen mucho que enseñar pero, en algunas oportunidades, estos excelentes profesionales carecen de formación pedagógica-didáctica para el desarrollo de su práctica docente. Por lo anterior, se define la interrogante como problema de investigación: ¿Cómo contribuir a solucionar las deficiencias que presenta el proceso de formación pedagógico-didáctico en los facilitadores, para que sea un proceso sistémico y pertinente? Con un objetivo fundamental que se refiere a Diseñar una Estrategia Metodológica para el desarrollo del proceso de formación pedagógico-didáctico para facilitadores. Guiando este proceso investigativo se generaron algunas preguntas y tareas científicas utilizando los métodos teóricos, empíricos y estadísticos permitiendo estos el desarrollo de las tareas de investigación. En tal sentido dicha investigación está sustentada teóricamente por Hernández y Sancho (1996:25) plantean que el tener conocimiento sobre un área específica, ser especialista o estudioso de una disciplina no implica de manera automática que pueda convertirse en enseñante con garantías de éxito. Y señalan que "saber la materia que se va a impartir, si bien es absolutamente necesario, no es condición suficiente para lograr o propiciar el aprendizaje del alumnado". Concluyendo preliminarmente la solución a este problema es la aplicación de estrategias pedagógicas- didácticas por parte de los facilitadores.
\end{abstract}

Descriptores: Proceso de formación pedagógico-didáctica; Formación Integral; Estrategia metodológica; Desempeño docente; Facilitadores. 


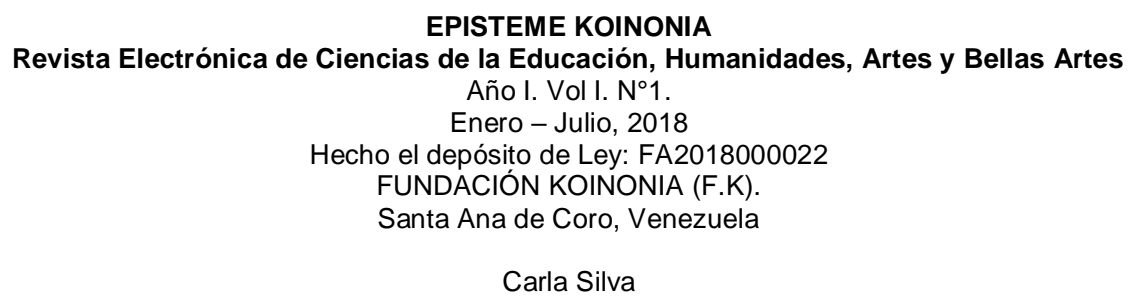

\begin{abstract}
The main objective of this work has been the elaboration of a methodological strategy for the development of the pedagogical-didactic training process for facilitators, starting from that the Universities intend to link to their staff of professors to people who stand out in their professional exercise and that, Therefore, considers that they have much to teach, but in some cases, these excellent professionals lack pedagogical-didactic training for the development of their teaching practice. For the above, the question is defined as a research problem: How to contribute to solve the deficiencies presented by the process of pedagogic-didactic training in the facilitators, so that it is a systemic and relevant process? With a fundamental objective that refers to designing a Methodological Strategy for the development of the pedagogic-didactic training process for facilitators. Guiding this research process were generated some questions and scientific tasks using the theoretical, empirical and statistical methods allowing these the development of research tasks. In this sense, this research is theoretically supported by Hernández and Sancho (1996: 25), argue that having knowledge about a specific area, being a specialist or a student of a discipline does not automatically imply that he can become a teacher with guarantees of success. And they point out that "knowing the subject to be taught, while absolutely necessary, is not a sufficient condition to achieve or foster student learning." Concluding preliminarily the solution to this problem is the application of pedagogicaldidactic strategies by the facilitators.
\end{abstract}

Descriptors: Pedagogical-didactic training process; Integral training; Methodological strategy; Teaching performance; Facilitators.

\title{
DESARROLLO
}

En el proceso de formación del docente o facilitador universitario, se puede expresar a través de la categoría pedagógico - didáctico estando estrechamente relacionado con los resultados finales que se logran al término del mismo; por lo que es posible identificarlos a través de los componentes de calidad del currículo de formación, a través de las siguientes categorías: Competencias didácticas; Dominio y transferencia de conocimientos; Comunicación interpersonal colectiva e individual y Autogestión personal y profesional.

En este sentido, el proceso de formación pedagógico-didáctico de los facilitadores debe permitir desarrollar competencias relacionadas con el desarrollo personal e inteligible, a través de las distintas actividades de enseñanza, que permiten un desempeño docente 


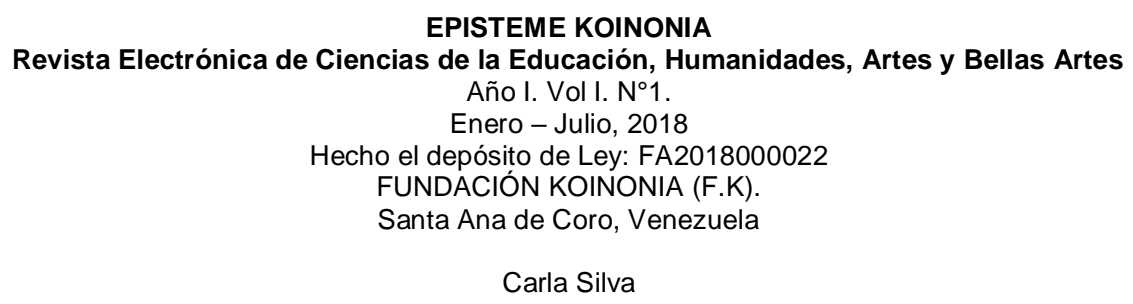

de constante perfeccionamiento y de realización personal. No cabe duda, que estas competencias tienen relación con la autoestima, la seguridad personal, la autoevaluación, la creatividad, la sensibilidad al cambio, la capacidad de innovación, la toma de decisiones y la resolución de problemas educativos.

Por otra parte, es común que los facilitadores de instituciones de educación secundaria sean Licenciados en un área específica, es decir, personas que voluntariamente decidieron estudiar para ser profesores. Con los docentes universitarios, ocurre algo diferente ya que, por lo general la universidad pretende vincular a su plantilla de catedráticos a aquellas personas que se destacan en su ejercicio profesional y que, por ende consideran que tienen mucho que enseñar, pero en algunas oportunidades, estos excelentes profesionales carecen de una formación pedagógica- didáctica para el desarrollo de su práctica docente.

De tal manera, que se genera la problemática que afecta la enseñanza del estudiante, ya que los que ejercen como docentes de las asignaturas son ingenieros en su pensum de formación profesional no tienen asignaturas curriculares orientadas a formarlos como docentes facilitadores académicamente.

Aunado, a ello los tecnológicos y universidades en general; por la carencia de profesionales en las áreas de matemáticas, química, física, biología, resistencia de los materiales, diseños estructurales, presupuestos de obras civiles, análisis de precios unitarios, cómputos métricos; otras materias; emplean en su plantilla profesoral a estos docentes para que impartan o faciliten los contenidos programáticos que tienen que ver con la formación en los niveles básicos y avanzados de la carrera de ingenieros.

La expresión de esta Situación polémica se evidencia repetitivamente, cuando estos profesionales ejercen a través de un contrato por horas la facilitación de una asignatura; sin ni siquiera una capacitación que contemple un componente o estrategias sencillas que les permita ser efectivos en la facilitación de los contenidos correspondientes a cada nivel, Asimismo, se destaca que estos profesionales de la ingeniería no manejan estrategias didácticas y por lo tanto, presentan deficiencias a nivel pedagógico como 


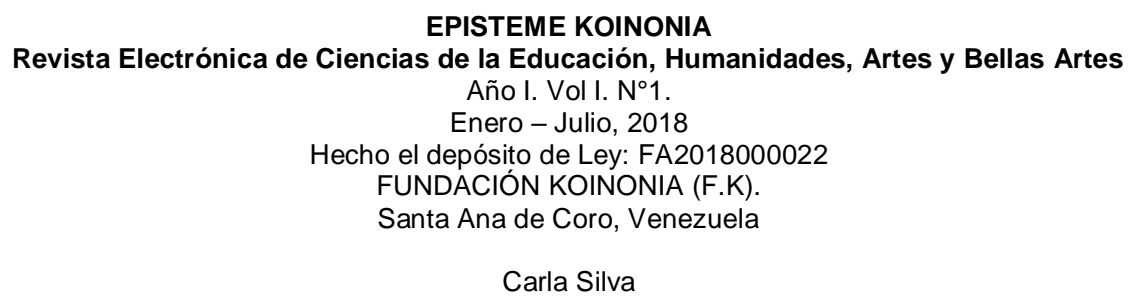

docentes facilitadores de las diferentes unidades de formación que conforman los pensum.

Como alternativa, a mejorar y transformar la educación en el Ministerio del Poder popular para la Educación en la revista científica Endurece (2007) sostiene que: "El Programa Nacional de Formación de Educadores, se convierte en una respuesta del Estado Venezolano a la creciente demanda del país para atender y promover la prosperidad y el bienestar y, con ello, saldar la deuda social acumulada por muchos años. Al instituirse la educación como derecho humano, ratifica su carácter público, de acceso libre a quien la necesita, convirtiéndose en función indeclinable y de máximo interés para el Estado. El Programa Nacional de Formación de Educadores pretende garantizar el cumplimiento de este derecho formando al Educador que en representación del Estado, asumirá el reto de guiar el proceso formativo con las características planteadas en los artículos 102 y 103 de la Carta Magna: Educador con un pensamiento Bolivariano, culto, portador de valores que dignifican al ciudadano venezolano, latinoamericano, caribeño y del mundo, con alto sentido de los valores humanos sociales, elementos contenidos en el artículo 104 de la Constitución y declarados como parte del Programa de Formación. (p.s/n)

Es por lo antes expresado que se pretende contribuir a la solución del problema, con un proceso de formación pedagógico-didáctico de los facilitadores, lo anteriormente expresado genera un Objetivo de investigación: Diseñar una Estrategia Metodológica para el desarrollo del proceso de formación pedagógico-didáctico de los facilitadores del Programa Nacional de Formación de Ingeniería en la Universidad Politécnica Territorial "José Félix Ribas" Barinas. Con Lineamientos Curriculares para Programas Nacionales de Formación, constituyen una acción fundamental en el marco de las Políticas de Educación Universitaria, pues serán la base tanto para el diseño y rediseño, como para la evaluación de los diseños curriculares. Según Gaceta Oficial No. 5987 (2010).

Es por ello y debido a la crecida vertiginosa en la creación de universidades los ingenieros que haciendo uso de sus multihabilidades, se dedican a trabajar como formadores en el programa nacional de formación de ingeniería en construcción civil en 


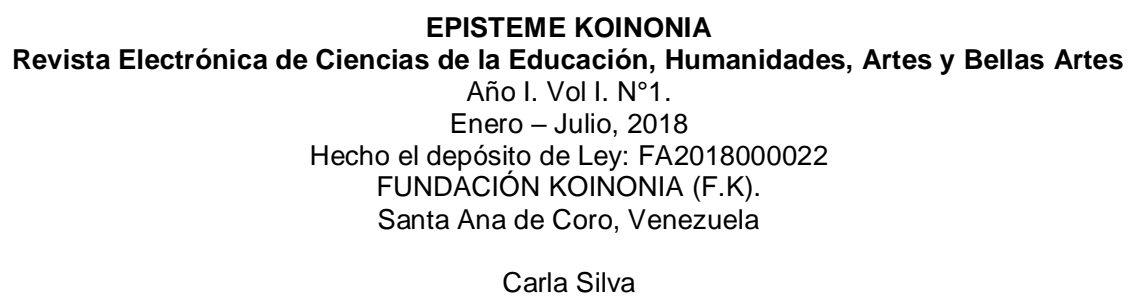

la Universidad Politécnica Territorial "José Félix Ribas", ubicada en Barinas, Estado Barinas, en la que los ingenieros de las diferentes cátedras de ingeniería civil, carecen de formación pedagógica-didáctica que les permitan desempeñarse de forma rendidora, es decir, que aun cuando poseen excelentes conocimientos y experiencia en su profesión como tal, al momento de transmitir los conocimientos en su función docente son confrontados debido a que hay situaciones en que el conocimiento se hace difícil hacerlo entender o llegar a los estudiantes, lo cual se convierte tanto para docentes como estudiantes en una situación estresante, motivado a que por la deficiencia de estrategias para hacer llegar el conocimiento de manera efectiva, muchos de los estudiantes son reprobados trayendo como consecuencia, quejas, insatisfacciones, descalificaciones del profesorado y por supuesto, esto crea malestar en los docentes ingenieros.

De allí, se genera la inquietud de crear una estrategia de formación pedagógica-didáctica que permita solventar las dudas y desarrollar de manera pertinente temas concernientes a la pedagogía y didáctica que los facilitadores desconocen ya que han sido formados en áreas distintas a la educación. A este respecto, Hernández y Sancho (1996:25) plantean que el tener conocimiento sobre un área específica, ser especialista o estudioso de una disciplina no implica de manera automática que pueda convertirse en enseñante con garantías de éxito. Y señalan que "saber la materia que se va a impartir, si bien es absolutamente necesario, no es condición suficiente para lograr o propiciar el aprendizaje del alumnado". Para ello, es necesario contar, entre otros aspectos, con una visión acerca de los modelos y estrategias didácticas, las teorías del desarrollo evolutivo, los diferentes estilos de aprendizaje y la influencia del entorno cultural y social para el desarrollo cognitivo de los alumnos.

Al respecto, la autora toma a continuación antecedentes de trabajos investigativos que permiten ampliar la descripción del problema e integrar el análisis de los hechos conocidos ayudando a la orientación de la búsqueda de otros datos relevantes:

Regno (2011) en su investigación realizada: estrategias de enseñanza del profesor en el aula de nivel superior. Desafíos para la formación docente. El propósito de esta 


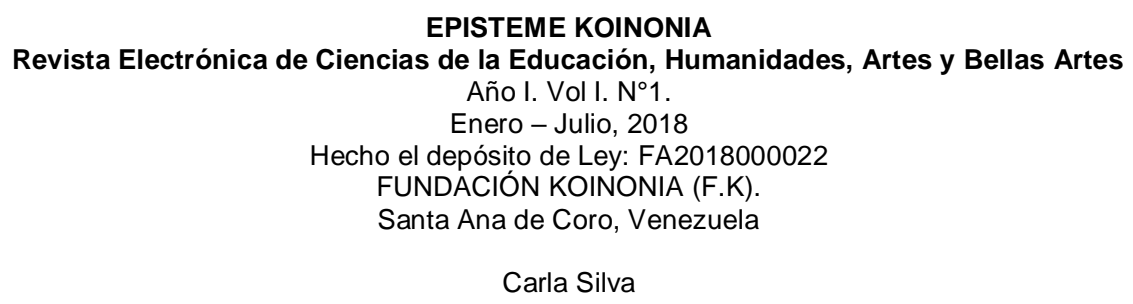

investigación es identificar, conocer y caracterizar las diversas estrategias de enseñanza que desarrolla el profesor en el aula de nivel superior universitario de la facultad de filosofía y letras de la universidad de Buenos Aires. De acuerdo a los objetivos de este trabajo de investigación, se podrían plantear a partir de los casos estudiados y avanzando en unas reflexiones más amplias algunos desafíos y propuestas básicas de mejora para la formación pedagógico-didáctica de los docentes: En principio, se puede crear conciencia a través de la Secretarías Pedagógicas institucionales o de figuras académicas reconocidas acerca de la importancia de promover acciones de asesoría y capacitación pedagógico-didáctica permanente a los profesores a través de diversas vías: la cursada de la Carrera Docente de la propia institución o de cursos externos (de instituciones estatales o privadas reconocidas y apreciadas a tal efecto), la participación en Congresos didácticos de la especialidad, la lectura de bibliografía y publicaciones didácticas del nivel superior (revistas especializadas, boletines temáticos, entre otros.), intercambios con profesores de otras instituciones, la participación en listas electrónicas de discusión temática docente sobre estos temas, (a través de Internet).

Este trabajo demuestra, que el docente para el conocimiento, un buen manejo y dominio de las estrategias de enseñanza y aprendizaje debe estar en constante preparación y actualización, que le permitan ir adaptando los contenidos académicos a acciones educativas factibles para un aprendizaje significativo de sus estudiantes.

Así mismo, Cardona (2004), realizó una investigación titulada: diseño del plan de formación docente en estrategias didácticas para el aprendizaje significativo en la institución universitaria Salazar y herrera, Medellín. El presente trabajo de investigación es el producto de la búsqueda incesante de querer implementar y mejorar nuevas estrategias didácticas que ayuden en la optimización de la labor docente. Donde se concluye, que las estrategias de enseñanza son utilizadas intencional o flexiblemente por el agente de enseñanza. Algunas de tales estrategias pueden emplearse antes de la situación de enseñanza, para activar el conocimiento previo o para tender puentes entre este último y el nuevo, (por ejemplo, los organizadores previos a los objetivos); otras en 


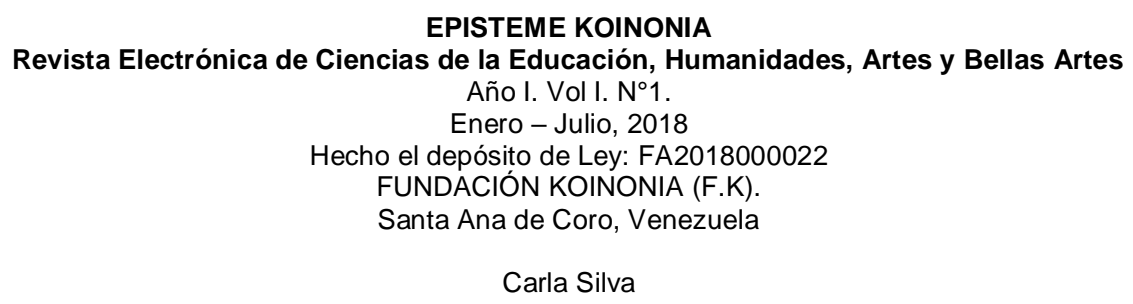

cambio llegan a utilizarse durante la situación de enseñanza para favorecer la atención, codificación y/o el procedimiento profundo de la información (las preguntas intercaladas, las señalizaciones); y otras más son útiles preferentemente al término de la situación de enseñanza, para reforzar el aprendizaje de la información nueva ( el resumen). Incluso ciertas estrategias como los mapas conceptuales, pueden emplearse en cualquier momento de la enseñanza.

Esta investigación demuestra, que existe una gran variedad de estrategias de enseñanza que puede ser utilizada por el docente al inicio, durante y al finalizar su clase, obteniendo con la implementación de las mismas un aprendizaje significativo, para lo cual sólo debe determinar y planificar las estrategias más convenientes tomando en cuenta el contenido a explicar, así como su contexto.

En tal sentido Avendaño, L (2008), en su estudio titulado: estrategias de enseñanza en la asignatura estudios de la naturaleza (propuesta de un plan de capacitación docente) tuvo como objetivo: diseñar un plan de capacitación docente en relación con las estrategias de enseñanza. Y en el cual concluyo: los docentes no poseen una definición clara de las estrategias de enseñanza, bien sea desde un punto general o específico. Del mismo modo en su mayoría no conocen la finalidad y uso de las estrategias de enseñanza, así como tampoco distinguen entre las estrategias de enseñanza y de aprendizaje, por lo tanto no emplean las estrategias en correspondencia con los momentos de la clase. Se pone de manifiesto las carencias de los docentes en cuanto a estrategias de enseñanza de las ciencias naturales, donde ninguno de ellos emplea las actividades experimentales para desarrollar los contenidos prácticos.

Esta investigación nos ayuda a comprender que los docentes no fomentan la construcción del conocimiento a través de la interacción activa de los estudiantes con los contenidos de la asignatura; no realizan actividades experimentales y prácticas que conduzcan a interactuar a los estudiantes con la realidad, promoviendo con esto el desequilibrio hacia la formación y transformación del aprendizaje de los estudiantes, ya que si no están enfocados que es una herramienta de enseñanza y el efecto de esta, no 


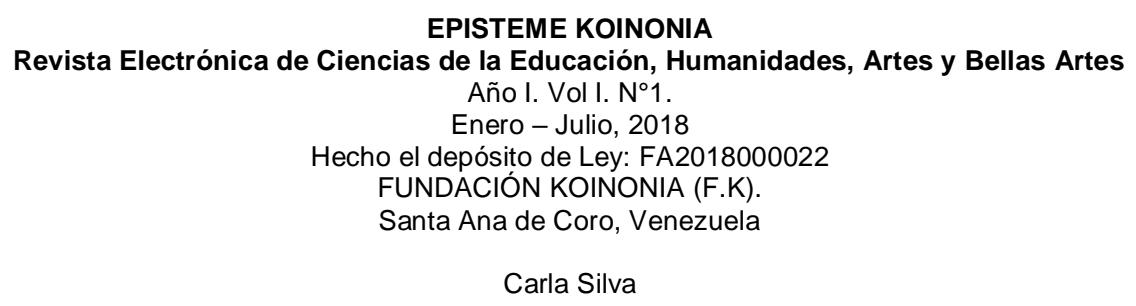

estamos ubicados hacia el crecimiento del estudiante, sino en el decrecimiento y así una mala concepción de lo que es enseñar.

Por otra parte Acosta (2005), en su investigación. Diagnóstico del desempeño docente del personal que labora en el área de investigación de la UPEL. Llegó a la conclusión de que la población docente estudiada en su gran mayoría posee títulos en maestrías, doctorados y otras especialidades, lo que indica que han recibido suficiente formación académica $\mathrm{y}$ en ese sentido tienes madurez y experiencia para comprender la importancia de su capacitación en el área e investigación. De igual manera los docentes manifiestan su voluntad de participar en jornadas académicas que le ayudase a mejorar en su desempeño y por ende en mejorar el rendimiento académico de sus estudiantes. Esta investigación representa un gran aporte en cuanto a la importancia de la estructuras jerárquica, ya que solo es posible llegar a una excelencia académica a través de una buena formación docente y de la disposición que esto manifiesten a la hora de aportar a cambios característico de los nuevos tiempos.

La formación del Profesorado es uno de los puntos que debe ocupar un lugar fundamental en la construcción del currículo, en la actualidad existe una mayor conciencia de lo que significa; bien pueden existir las reformas en las estructuras de lo que se debe enseñar, pero si esto se haya desvinculado de lo que el profesor debe conocer y de la dignificación de su labor, estas modificaciones por más bien intencionadas y fundamentadas que sean, se quedarán en la letra muerta o, peor aún, generar resultados antagónicos a lo planeado. Los profesores son los interlocutores decisivos más importantes de los cambios educativos; son los agentes del currículum, son sujetos del cambio y la renovación pedagógica.

De lo anterior se derivan dos conceptos fundamentales que son la profesionalización docente y el desempeño profesional de la docencia, los cuales se profundizaran en el marco contextual; el primero se refiere a las diversas decisiones políticas, sociales y formativas que conforman la profesión docente, su valor social e intelectual, lo cual se traduce en aspectos y condiciones como el salario, conocimientos y competencia para el 


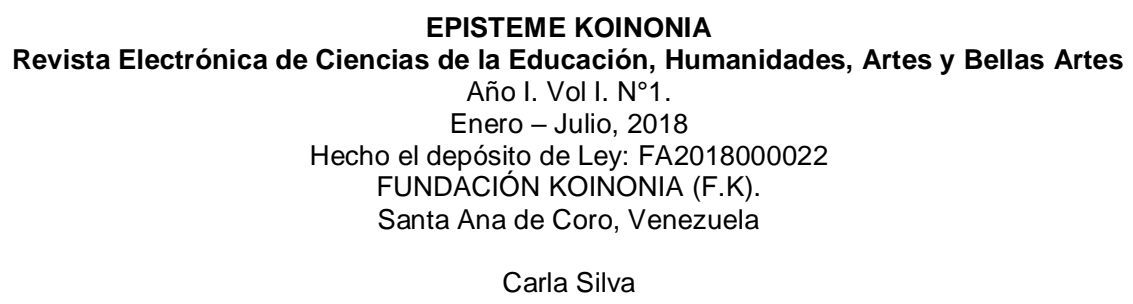

acceso a la profesión, estructuras organizativas, contextos profesionales y condiciones de trabajo. Por otra parte el término de desempeño profesional se refiere al carácter y la calidad del que hacer y práctica docentes de acuerdo con ciertos criterios y pautas que se valoran como un buen desempeño. Sin embargo, las prácticas docentes destinan poca reflexión y análisis respecto a estos aspectos y esto genera que se repitan los procedimientos convencionales fincados en el supuesto de que el aprendizaje es ante todo el simple registro y repetición de la información vertida por el maestro o en el libro. Formación Permanente e integral no será posible o, si lo es, sus efectos serán poco perdurables, si los profesores experimentados no entran a formar parte de esta dinámica. Al respecto, Moreno (2006) señala: un profesor experimentado "es aquel que ha superado la etapa de profesor novel, y posee una experiencia docente suficiente para ejercer una docencia más centrada y madura, de manera que sus necesidades de formación son distintas a las de los primeros" (p. 35).

No es extraño conseguir que profesores experimentados no se asocien a planes de mejora docente. Por lo general, con el paso del tiempo, muchos de los problemas, agobios y dudas se van superando de mejor o peor manera y cada profesor se hace con unas rutinas que juzga adecuadas. Es evidente que la práctica es un elemento fundamental de aprendizaje, pero siempre y cuando se cumpla la condición de que se trate de una práctica reflexiva. Sólo desde los procesos reflexivos acerca de la acción es posible generar observaciones que permitan reorientar la acción hacia direcciones más adecuadas. Para que esta reflexión tenga un carácter más sistemático y productivo parece aconsejable que se ofrezca a los profesores la oportunidad de ponerse en contacto con experiencias innovadoras y se les dote de elementos teórico - prácticos que orienten esa reflexión.

Por consiguiente, Navarrete (2006), comenta que "para aprender a mejorar su práctica profesional, el docente ha de contrastar sus teorías previas con las evidencias de una reflexión rigurosa sobre su quehacer" (p. 17). 


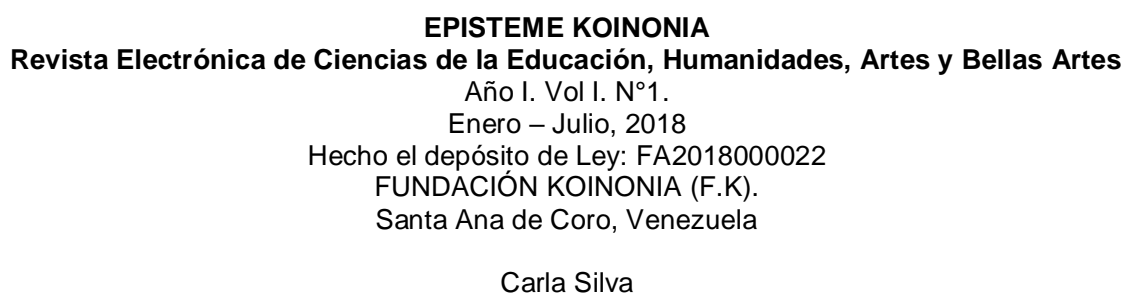

Las estrategias son acciones que tienden al logro de un objetivo. Según Kirby son medios de selección, combinación o planificación de las habilidades cognitivas ante una situación dada. El camino para adquirir destrezas.

De acuerdo a Vygotsky, citado por Mendoza (2001), Las estrategia metodológica constituyen, "formas con los que cuenta el estudiante y el docente para controlar los procesos de aprendizaje, así como la retención y el pensamiento".

Vygotsky dice además que la aplicación correcta de estrategia metodológica posibilita el manejo de una serie de habilidades, que permiten a la persona identificar una alternativa viable para superar una dificultad en la que no existen soluciones conocidas. Esta es la habilidad para resolver problemas y requiere del uso de todas las capacidades específicas del estudiante y de la aplicación de todas las estrategias posibles, solo de esta manera se conseguirán niveles de pensamiento más elevados y con un grado de complejidad cada vez mayor.

Regularidades encontradas como resultado del análisis de los instrumentos de diagnóstico.

Los formadores del PNF Construcción Civil de la Universidad Politécnica Territorial José Félix Ribas del estado Barinas civil carecen de preparaciones pedagógico-didácticas a través de las cuales puedan profundizar en la metodología de la clase.

Dentro del proceso enseñanza- aprendizaje existen dificultades por parte de los formadores para hacerse entender, y en los estudiantes, para entender a los formadores lo que les conlleva a reprobar las evaluaciones.

Se encontró que los formadores en el tiempo que llevan impartiendo clases están utilizando las mismas estrategias cada año, siendo estas las que ellos recibieron cuando fueron estudiantes.

Tanto facilitadores como estudiantes coinciden en que se trata de lograr la participación, estimulando e innovando la superación de dificultades de aprendizaje en los estudiantes. El proceso de formación pedagógico-didáctico de los formadores en la carrera de Ingeniería en construcción civil requiere ser rediseñado en función de sistematizarse 


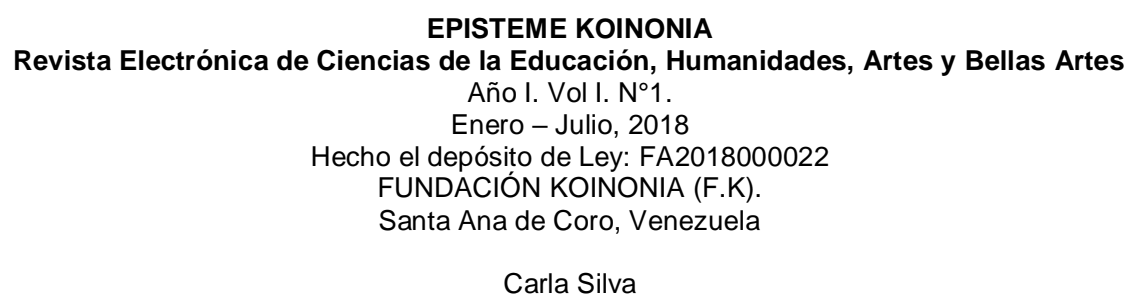

según las unidades de formación que se imparte, ya que estos manifiestan utilizar las mismas estrategias en todas las asignaturas.

\section{Propuesta para la formación pedagógica del profesorado universitario}

Se concibe la formación pedagógica del profesorado universitario como un proceso continuo que atendiendo a diferentes etapas organizadas en su práctica docente, facilitan iniciar, adiestrar, formar y perfeccionar a dichos profesores en el dominio de los contenidos de la pedagogía y la didáctica de la educación superior con el propósito de incidir en la calidad de la formación de los estudiantes lo que influye en la calidad de la educación universitaria.

La estrategia diseñada contiene la siguiente estructura:

III.1 Estructura de la estrategia:

\section{III.1.1. Introducción}

El diseño de la estrategia va dirigida a proporcionar a los facilitadores del Programa Nacional de Formación de Ingeniería en Construcción Civil, un proceso de formación pedagógica-didáctica que les permita direccionar el proceso de enseñanza aprendizaje en un contexto determinado.

III.1.2. Objetivo de la estrategia

Implementar en la práctica educativa el proceso de formación pedagógica-didáctica de los facilitadores del Programa Nacional de Formación de Ingeniería en Construcción Civil en la Universidad Politécnica Territorial "JOSÉ FÉLIX RIBAS". Estado Barinas. Venezuela.

III.2. Acciones de la estrategia

Las acciones diseñadas cumplen con operaciones donde su ejecución es asignada a actores en fechas determinadas durante el tiempo del año en estudio. 


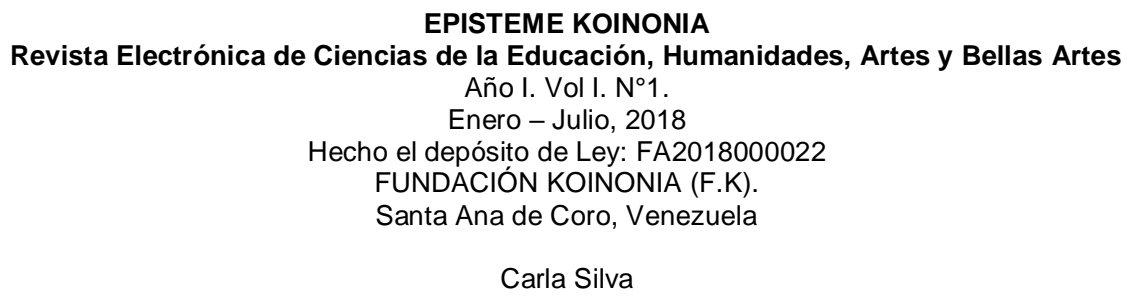

\section{Acciones de la estrategia}

\begin{tabular}{|c|c|c|c|}
\hline ACCIONES & OPERACIONES & FECHAS & PARTICIPAN \\
\hline $\begin{array}{l}\text { 1.Diseñar } \\
\text { programa de } \\
\text { formación } \\
\text { pedagógica- } \\
\text { didáctica para los } \\
\text { facilitadores a partir } \\
\text { de las 4 etapas } \\
\text { concebidas } \\
\end{array}$ & $\begin{array}{l}\text { 1- Revisar el diagnostico } \\
\text { aplicado a los facilitadores y } \\
\text { estudiantes. } \\
\text { 2- Diseñar el programa. } \\
\text { 3- Reunir al colectivo de } \\
\text { facilitadores para iniciar el } \\
\text { proceso de formación }\end{array}$ & $\begin{array}{l}\text { Enero- } \\
\text { marzo }\end{array}$ & facilitadores \\
\hline $\begin{array}{l}\text { 2.Impartir el } \\
\text { programa de } \\
\text { formación } \\
\text { diseñado desde las } \\
4 \text { etapas descritas }\end{array}$ & $\begin{array}{l}\text { 1. Programar los tiempos para } \\
\text { impartir el programa de } \\
\text { capacitación. } \\
\text { 2. Impartir el programa. } \\
\text { 3. Evaluar el programa }\end{array}$ & $\begin{array}{l}\text { Abril } \\
\text { mayo }\end{array}$ & facilitadores \\
\hline $\begin{array}{llr}\text { 3. Evaluar } & & \text { los } \\
\text { resultados } & \text { de } & \text { la } \\
\text { formación } & \text { y } & \text { el } \\
\text { programa } & & \end{array}$ & $\begin{array}{l}1 \text { Aplicar diagnósticos de } \\
\text { satisfacción de los docentes a } \\
\text { cerca del proceso de } \\
\text { formación. } \\
\text { 2. Hacer evaluación de } \\
\text { impacto del programa }\end{array}$ & $\begin{array}{l}\text { Junio } \\
\text { julio }\end{array}$ & facilitadores \\
\hline
\end{tabular}

A partir de la revisión del diagnóstico aplicado a los docentes y estudiantes, y sobre las bases teóricas, los métodos, medios y formas en la que se basa esta estrategia, se reunirá al colectivo de diseñadores para elaborar la misma. Entre los métodos que se priorizarán están aquellos que por las características del proceso de enseñanza aprendizaje de la Ingeniería en Construcción Civil respondan a las necesidades de formación detectada; según el grado de participación: el método expositivo, la elaboración conjunta, trabajo independiente; desde la fuente de obtención del conocimiento: verbal, visual y práctico; de acuerdo al grado de dominio esperado de los estudiantes: reproductivo, productivo, creativo; los que estimulan la actividad productiva: heurístico, investigativo; y de acuerdo a la lógica de desarrollo del proceso de enseñanza: introducción, desarrollo, dominio, sistematización y evaluación de contenido.

Con respecto a los medios como vehículo mediante el cual se manifiesta el método, se espera ir desde la realidad objetiva al pensamiento abstracto y de este a la práctica donde 


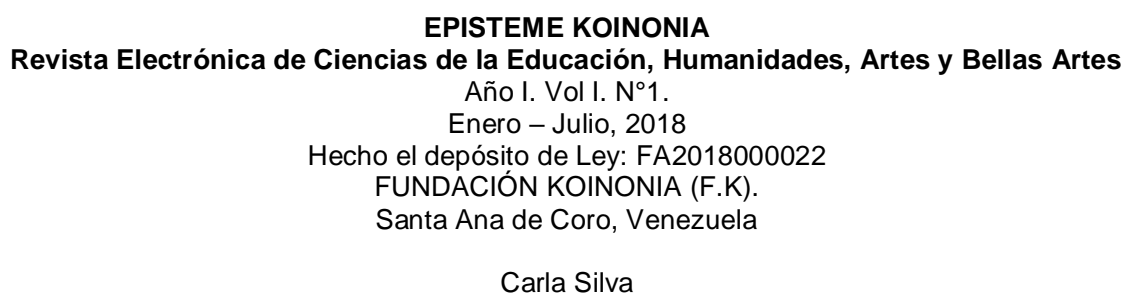

el estudiante evidencie la experiencia, el análisis y la comprensión de la experiencia pedagógica didáctica; a partir del uso de los fundamentos fisiológicos de los medios de enseñanza, se logra el desarrollo del pensamiento; desde la utilización de los fundamentos psicológicos se logra elevar los niveles de motivación por el aprendizaje, los medios de enseñanza permitirán elevar la efectividad del proceso de enseñanza aprendizaje logrando racionalizar los esfuerzos de los facilitadores y estudiantes, dinamizando el proceso de enseñanza aprendizaje, propiciando el trabajo en grupos. Utilizarán medios audiovisuales, los manipulativos, textuales o impresos e informáticos. La forma como organización y orden que adopta el proceso para alcanzar el objetivo tiene dimensión espacial y dimensión temporal, estará sujeta a la cantidad de participantes y a la disponibilidad en el tiempo de preparación que los docentes involucrados en esta estrategia dispongan y acuerden para capacitarse en el programa diseñado.

La evaluación de los aprendizajes se fundamentará en el uso de técnicas informales, semi-formales y formales. Como técnicas informales: observación de las actividades realizadas por los estudiantes, exploración a través de preguntas formuladas durante la clase. Técnicas semi-formales: Ejercicios y prácticas en clase, tareas, evaluación de portafolio. Técnicas formales: Pruebas prácticas, Escala de evaluación.

Se evaluará los resultados de la capacitación y el programa implementado efectuando un diagnóstico de satisfacción de los docentes acerca de la capacitación, a fin de verificar el logro de los objetivos propuestos; por último se hará evaluación de impacto del programa a través del diagnóstico efectuado a los estudiantes y por medio de entrevista a las autoridades del programa, así mismo, lanni Gómez (2017), señala que el docente debe formarse para el rol de asesor investigador para propiciar la transformación educativa, mientras Romero \& Villasmil (2017), señalan la necesidad de repensar la formación del docente en la formación universitaria con la finalidad de promover un enfoque transformacional educativo con la finalidad de contar con facilitadores en concordancia con las expectativas de la educación centrada en la investigación. 


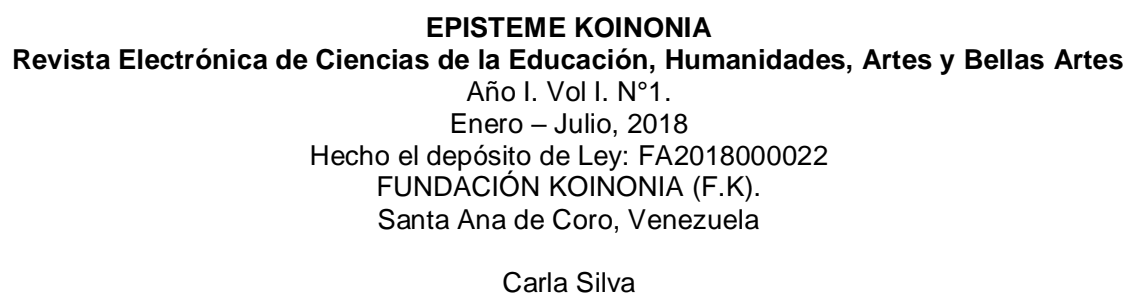

\section{REFERENCIAS CONSULTADAS}

1. Acosta (2005). Diagnóstico del desempeño docente del personal que labora en el área de investigación de la Upel. Trabajo de grado para optar al título de licenciado. Universidad Pedagógica Experimental Simón Bolívar, Sucre-Venezuela.

2. Avedañado, L (2008). Estrategias de enseñanza en la asignatura estudios de la naturaleza (propuesta de un plan de capacitación docente). Trabajo de grado para optar al título de licenciado. Universidad de los andes, Venezuela.

3. Cardona, J. (2004). Diseño del plan de formación docente en estrategias didácticas para el aprendizaje significativo. [Revista en línea]. Universidad Salazar y herrera, Medellín. Disponible:http://tesis.udea.edu.co/dspace/bitstream/10495/189/1/DisenioPlanfor macionDocentesEstrategiasDidacticas.pdf.

4. Díaz, F Sancho y Hernández G. (1996). Docentes del siglo XXI; Editorial Mc GrawHill. Colombia. El aprendizaje significativo de David Paul Ausubel [Bases de datos en línea]. Consultado el 25 de junio del 2015 en:http//www.monografias.com/trabajo10/data7data.shtm/1997monografias.com.

s.a

5. Ianni Gómez., L. (2017). Miramda: Una propuesta educativa emergente desde la investigación. Revista Arbitrada Interdisciplinaria Koinonía, 2(3), 9-30.

Recuperado de http://fundacionkoinonia.com.ve/ojs/index.php/revistakoinonia/article/view/49/36

6. Moreno, M (2006). Estrategias de aprendizaje en estudiantes universitarias de primer año de la carrera educación parvularia. [Revista en línea]. Universidad Católica del Maule Chile. Disponible: http://www.psicologiacientifica.com/bv/psicologia-62-1-estrategias-deaprendizajeen-estudiantes-universitarias.html.

7. Navarrete, B. Chacón, M (2006). Prácticas profesionales en la formación docente: hacia un nuevo diario de ruta. Trabajo de grado para optar al título de licenciado. Estudio realizado en el estado Trujillo, Venezuela.

8. Poder Legislativo De Venezuela (2010) Gaceta Oficial No. 5987 Extraordinario. Caracas, Venezuela. Creación de la Universidad Politécnica Territorial del Estado Barinas. 


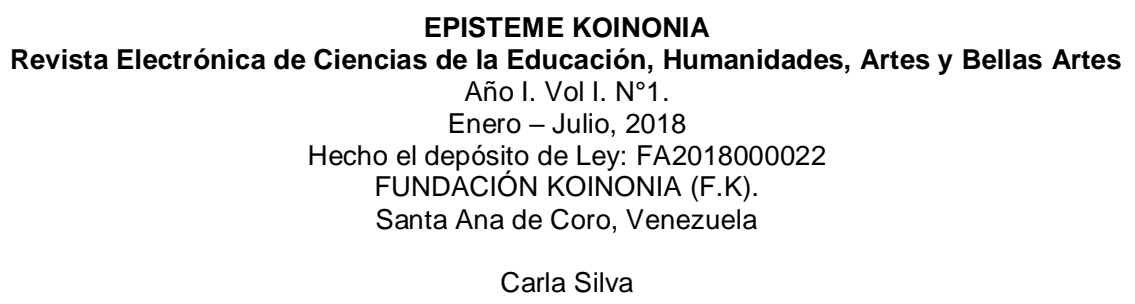

9. Regno, P. (2011). Estrategias de enseñanza del profesor en el aula de nivel superior. Desafíos para la formación docente. [Revista en línea]. Universidad de Buenos Aires. Disponible: http://www.mdp.edu.ar/humanidades/pedagogia/jornadas/jprof2011/comunicacio nes/ 013.pdf

10. Romero, M., \& Villasmil, J. (2017). Repensar la formación docente. Hacia el encuentro de una nueva perspectiva epistémica para su abordaje y resignificación. CIENCIAMATRIA, 3(5), 133-149. https://doi.org/10.35381/cm.v3i5.17

11. Vigotsky, Mendoza (2011), y Otros. "Psicología de una Perspectiva Científica "(Academia ADUNI). Lima 2001 Pág. 239 - 248. Leer más: http://www.monografias.com/trabajos59/estrategias-vigotsky/estrategiasvigotsky2.shtml\#ixzz4OIGDxIY3

\section{REFERENCES CONSULTED}

1. Acosta (2005). Diagnosis of the teaching performance of staff working in the research area of the Upel. Degree work to qualify for the bachelor's degree. Simón Bolívar Experimental Pedagogical University, Sucre-Venezuela.

2. Avedañado, $L$ (2008). Teaching strategies in the nature studies subject (proposal of a teacher training plan). Degree work to qualify for the bachelor's degree. University of the Andes, Venezuela.

3. Cardona, J. (2004). Design of the teacher training plan in teaching strategies for meaningful learning. [Online magazine]. Salazar y herrera University, Medellín. Available: http: //tesis.udea.edu.co/dspace/bitstream/10495/189/1/DisenioPlanformacionDocente sEstrategiasDidacticas.pdf.

4. Díaz, F Sancho and Hernández G. (1996). Teachers of the 21st century; Editorial Mc Graw-Hill. Colombia. The meaningful learning of David Paul Ausubel [Online databases]. Retrieved on June 25, 2015 at: http // www.monografias.com / trabajo10 / data7data.shtm / 1997monografias.com.s.a

5. Moreno, M (2006). Learning strategies in first year university students of the preschool education career. [Online magazine]. Catholic University of Maule Chile. Available: $\quad$ http://www.psicologiacientifica.com/bv/psicologia-62-1-estrategiasdeaprendizaje-en-estudiantes-universitarias.html. 


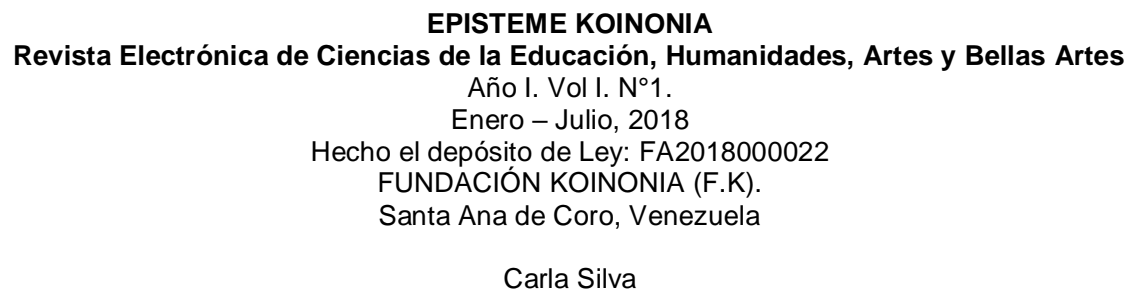

6. Navarrete, B. Chacón, M (2006). Professional practices in teacher training: towards a new route diary. Degree work to qualify for the bachelor's degree. Study conducted in Trujillo state, Venezuela.

7. Legislative Power of Venezuela (2010) Official Gazette No. 5987 Extraordinary. Caracas Venezuela. Creation of the Territorial Polytechnic University of the State Barinas.

8. Regno, P. (2011). Teaching strategies of the teacher in the upper level classroom. Challenges for teacher training. [Online magazine]. Buenos Aires' University. Available:

http://www.mdp.edu.ar/humanidades/pedagogia/jornadas/jprof2011/comunicacio nes/ 013.pdf

9. Vigotsky, Mendoza (2011), and Others. "Psychology of a Scientific Perspective" (ADUNI Academy). Lima 2001 Page 239 - 248. Read more: http://www.monografias.com/trabajos59/estrategias-vigotsky/estrategiasvigotsky2.shtml\#ixzz4OIGDxIY3

(C)2018 por los autores. Este artículo es de acceso abierto y distribuido según los términos y condiciones de la licencia Creative Commons Atribución-NoComercial-CompartirIgual 4.0 Internacional (CC BY-NC-SA 4.0) (https://creativecommons.org/licenses/by-nc-sa/4.0/). 